\title{
Elaboración de escenarios de prospectiva para asentamientos humanos urbanos y rurales, aplicado al municipio de Tela, Honduras
}

Claudia Nataly Mondragón Rivera y

Francisco Maza Vázquez

\section{Resumen}

Las características peculiares de las políticas de suelo de Centroamérica, la tenencia de la tierra que conforman sus territorios, los condicionantes de desarrollo urbano, su demografía, sus actividades económicas, infraestructurales y demás actores que intervienen en la ordenación del territorio exige de una metodología que proporcione un instrumento de ayuda en la toma de decisiones.

En este sentido, se presenta una metodología, aplicada al municipio de Tela, situado al norte de Honduras, que facilita la elaboración de escenarios de prospectiva para asentamientos humanos, en suelos urbano y rural, con el fin de poder planificar su crecimiento con garantías de bienestar. Ello nos ayudará a comprender cómo las acciones que hoy tomamos pueden influir en el futuro de los territorios.

Palabras clave: Desarrollo. Territorio. Ordenación. Asentamientos humanos.

\section{Abstract}

The peculiar characteristics of the Central American land policy, land tenure that makes their territories, the constraints of urban development, demographics, economic activities, infrastructure and other factors involved in regional planning require a methodology that provides an aid in decision making.

Here, we present a methodology, applied to the town of Tela, just north of Honduras, which facilitates the development of prospective scenarios for human settlements, urban and rural land, in order to plan their growth with guarantees of welfare. This will help us understand how the actions we take today can influence the future of the territories.

Keywords: Development. Territory. Management. Human settlements. 
Claudia Nataly Mondragón Rivera (naty_06hn@hotmail.com), Universidad Nacional Autónoma de Honduras. Francisco Maza Vázquez (francisco.maza@uah.es), Universidad de Alcalá. (España). 


\section{INTRODUCCIÓN}

Según la Real Academia Española, se entiende por prospectiva, (del lat. prospicere, mirar). ${ }^{1}$. adj. Que se refiere al futuro. ${ }^{2}$. f. Conjunto de análisis y estudios realizados con el fin de explorar o de predecir el futuro, en una determinada materia.

En los planes de ordenamiento territorial, la prospectiva es una fase metodológica intermedia entre el diagnóstico y la propuesta, y sirve para orientar la anterior considerando diferentes escenarios 1 futuros. Se trata de proyectar, a partir del diagnóstico, las variables más representativas y diseñar escenarios futuros; a saber, el tendencial, el óptimo y el intermedio o de consenso, entre otros.

La experiencia en el diseño de planes de ordenación del territorio (u ordenamiento territorial, término más usado en Latinoamérica2) y la lectura de informes, libros y otros relacionados con la materia, nos ha demostrado que la prospectiva se considera la fase más compleja y peor desarrollada.

Se pretende en este trabajo realizar un análisis de los precedentes de la prospectiva en planes de ordenamiento territorial. Para ello utilizaremos el siguiente MARCO CONCEPTUAL:

- La prospectiva como fase intermedia de la planificación territorial.

- La prospectiva según Gaston Berger.

- El modelo de Michel Godet. La escuela francesa.

- La escuela anglosajona.

- El modelo del Dr. Colino desarrollado para el valle de San Andrés (El Salvador); determinación de la capacidad de acogida poblacional de un territorio basada en los recursos naturales en un escenario de desarrollo sostenible.

- El modelo de prospectiva del Dr. Gómez Orea y su aplicación a algunos casos prácticos.

- Análisis de caso en Honduras:

\footnotetext{
${ }^{1}$ Imágenes o visiones.

${ }^{2}$ El trabajo de investigación se ha realizado en varios países de Centroamérica, en especial El Salvador, Honduras, Nicaragua y Costa Rica.
} 
- La prospectiva aplicada al análisis territorial del municipio de Tela, Honduras.

- La participación como herramienta básica para abordar la fase de prospectiva.

El municipio de Tela se encuentra en el departamento de Atlántida. Sus límites territoriales son: Al norte, con el mar Caribe; al sur, con el municipio de Yoro (departamento de Yoro); al este, con el municipio de Arizona (departamento de Atlántida); y al oeste, con el municipio de Puerto Cortés (departamento de Cortés). Se encuentra ubicado entre las coordenadas geográficas siguientes: $15^{\circ} 47^{\prime}$ latitud norte, $87^{\circ} 28^{\prime}$ longitud oeste.

Para el censo de 1974, las aldeas del municipio de Tela eran 30. Para el censo de 1988 y del 2001 se registran 76 aldeas. Los caseríos del municipio son 272 según datos del censo del 2001 y los cascos urbanos son 3 (Tela, Triunfo de la Cruz y Mezapa).

\section{HIPÓTESIS}

Esta metodología se considera un instrumento de ayuda en la toma de decisiones a través del conocimiento de las tendencias urbanas reales en Centroamérica, mediante el estudio de variables como los condicionantes al desarrollo urbano, demografía, actividades económicas. Con su aplicación se debe permitir la representación de diferentes imágenes del futuro que ayudarán a comprender cómo las acciones que hoy tomamos pueden influir en el futuro de los territorios.

La metodología constituye una contribución valiosa a las políticas de suelo urbano de Centroamérica, ya que sus lineamientos se acoplarán a la realidad existente en la región, una realidad difícil pero mejorable a través de políticas territoriales flexibles y coherentes con las características del territorio.

\section{OBJETIVO GENERAL}

Se pretende elaborar escenarios de prospectiva, mediante una metodología aplicable a la realidad hondureña y centroamericana, teniendo en cuenta diferentes análisis y puntos de partida, entre ellos las autómatas celulares y una evaluación multicriterio basado en el análisis de la capacidad de acogida del territorio. 


\section{Objetivos específicos}

Se persiguen los siguientes objetivos específicos:

- Implementar un modelo metodológico para diseñar los escenarios o sistemas territoriales futuros, aplicable a los asentamientos humanos urbanos y rurales en Honduras.

- Desarrollar la forma de representar los escenarios en mapas, de manera fácilmente comprensible por los actores o agentes socioeconómicos y por la ciudadanía, que son los "clientes" finales del plan.

\section{Contribución y metodología}

La principal contribución de esta investigación es el desarrollo de una metodología útil, transparente y flexible para la fase de prospectiva en planes de ordenamiento territorial de Centroamérica, capaz de integrar las variables estratégicas de los subsistemas territoriales, así como proyectar las mismas mediante algoritmos matemáticos e interpretarlos a través del consenso entre expertos y actores territoriales. La metodología se ha aplicado a varios planes de la región centroamericana por lo que está suficientemente contrastada y su ejecución es relativamente sencilla, con la existencia de algunos datos, una mínima experiencia y conocimiento territorial.

\section{LA JERARQUIZACIÓN: EL SISTEMA DE ASENTAMIENTOS HUMANOS}

\section{URBANOS Y RURALES}

Se seleccionan los asentamientos humanos con población mayor a 500 habitantes y los mismos se jerarquizan siguiendo diferentes criterios:

Criterio demográfico: A este criterio se le ha dado el mayor peso dentro del análisis. El criterio utilizado principalmente ha sido el correspondiente a los datos de población 2001, para los asentamientos ubicados en el municipio. Definiendo oficialmente cinco categorías, para este análisis se ha propuesto una valoración de 0 a 70 .

Puntuación:

- 70: población mayor a 10.000 habitantes

- 56: población con un rango de 5.000 y 10.000 habitantes

- 42: población con un rango de 1.000 y 5.000 habitantes

- 28: población con un rango de 500 y 1.000 habitantes

- 14: población menor a 500 habitantes. 
Imagen urbana: La imagen urbana como criterio para la categorización de los asentamientos humanos, se basó en la correcta imagen urbana así como la coherencia en la trama urbana, destacando aspectos como accesibilidad, espacio público, áreas verdes. Para este análisis se ha propuesto una valoración de 0 a 10.

Puntuación

- 10: Asentamiento con una satisfactoria imagen y coherencia urbana.

- 5: Asentamiento con una media imagen urbana y coherencia en la trama de la misma.

- $\quad$ : Asentamiento con deficiencia en la imagen urbana, así con poca coherencia en la imagen de la misma.

Accesibilidad: Relación de los asentamientos con las vías de comunicación según la categoría de vías principales, vías secundarias, vías terciarias,

Puntuación

- 10: con acceso a vías principales

- 5: Con acceso a vías secundarias

- 0: acceso a vías vecinales y de herradura

Equipamientos y servicios: Las actividades comerciales y servicios en un núcleo poblacional es un factor importante en la categorización de los asentamientos humanos, ya que estos se rencuentran directamente relacionado con la economía local y regional.

Puntuación

- 10: Asentamiento con una satisfactoria actividad comercial y con un área de influencia para brindar servicios a los habitantes de comunidades vecinas.

- 5: Asentamiento con una mediana actividad comercial y con la suficiente capacidad de servicios para satisfacer a sus habitantes.

- $\quad$ : Asentamiento con una poca actividad comercial, y poca capacidad de servicios.

\section{CLASIFICACIÓN POTENCIAL DE LOS ASENTAMIENTOS HUMANOS}

SEGÚN LOS LIMITANTES AL DESARROLLO URBANO

Los asentamientos humanos sostenibles dependen de la creación de un 
entorno mejor para la salud y el bienestar de las personas que allí habitan, que mejore sus condiciones de vida y reduzca las disparidades en su calidad de vida. Entre los factores a tener en cuenta para crear asentamientos adecuados destacamos las amenazas y los riesgos naturales (que afectan directamente a la población), aparte de proteger las áreas con un alto mérito de conservación ya establecido anteriormente. Los asentamientos humanos, serán clasificados según sus áreas inundables, áreas con pendientes elevadas, áreas protegidas, y méritos de conservación. La misma puntuación establecerá cuales son los asentamientos humanos a incentivar, a consolidar y a desincentivar, en función de los limitantes al desarrollo urbano.

\section{VARIABLES DEMOGRÁFICAS}

La prospectiva demográfica para el municipio es el conjunto de resultados relativos a la evolución de la población futura, provenientes de cálculos estadísticos, que se apoyan en el comportamiento pasado y reciente de las variables determinantes de su dinámica, la natalidad, la mortalidad y las migraciones y los supuestos adoptados de evolución de estas variables, hasta el final del período de proyección. La proyección demográfica nos ayuda a calcular la dotación futura de servicios básicos (educación, salud, agua, etc.) datos:

Para la presente investigación se ha utilizado las siguientes fuentes de

- Censos nacionales de población 1988, 2001.

- Estimaciones oficiales de población para el municipio de Tela.

La prospectiva demográfica, que sirve para determinar los escenarios poblacionales, permite establecer el marco de referencia de una de las variables básicas de todo proceso de planificación espacial. Para la realización de esta prospectiva se establecen dos fases secuenciales:

- En la primera, y a través de las correspondientes proyecciones de población, se obtienen los valores poblacionales para el municipio, a partir de las proyecciones oficiales. Lo que se obtiene es el dato para el conjunto de ámbito territorial.

- Una segunda fase, es la del establecimiento de los escenarios demográfico-territoriales. Para la determinación de los mismos se parte no solo de la variable poblacional, sino que se estiman a partir de los objetivos planteados en los diferentes apartados del plan y de los determinantes que las actuaciones finales en materias productivas, in- 
fraestructurales y urbanísticas implican a nivel de desarrollo territorial en el municipio.

Según lo anterior se establecen los siguientes:

- Proyección de la población del municipio: Se calcula a partir de las proyecciones oficiales de población, lo que se denomina población tendencial, y a partir de la misma se establecen dos escenarios: "el conservador", que parte de la dinámica actual de población, y "el desarrollista". Considerando que existirán una serie de acciones territoriales que impactarán atrayendo población al territorio; cabe señalar que la cantidad de población, en los tres escenarios es la misma, lo que varía es la distribución de la población dentro del municipio.

- Escenarios demográficos, población urbana y rural; a partir del escenario tendencial. Ante la incertidumbre que plantea el futuro y considerando las variables anteriores, debemos considerar la hipótesis del escenario más desfavorable y planificar el territorio y los recursos naturales: suelo, agua, servicios básicos, etc. En función de lo anterior, distribuimos la población urbana y rural considerando tres casos:

a) Escenario tendencial: se mantiene la distribución actual y el porcentaje de urbano vs. rural en el municipio, no varía considerablemente según al actual.

b) Escenario óptimo: el proceso de urbanización funciona de forma acelerada en las zonas y asentamientos que cuentan con las condiciones territoriales idóneas.

c) Escenario intermedio: MTF utilizando el $40 \%$ del modelo tendencial y el $60 \%$ del modelo óptimo, dejando así un análisis equilibrado.

Cabe señalar que sobre la proyección demográfica (tendencial) de población en términos absolutos es difícil incidir, ya que ello depende de muchos factores internos y externos (voluntad política, impacto de las acciones, acciones impredecibles como migraciones, riesgos naturales, inversiones no previstas, etc.) pero sí es posible dirigir la distribución de la población urbana y rural, a través de dotación de suelos y una gestión eficaz del territorio.

Las definiciones y categorías expuestas nos sitúan en el estado del arte conceptual, cuya aplicación desarrollamos a continuación en el área de estudio: municipio de Tela. 


\section{DIAGNÓSTICO: SISTEMA DE ASENTAMIENTOS HUMANOS MUNICIPIO DE TELA}

Los principales asentamientos del municipio de Tela fueron evaluados para determinar el grado de relevancia municipal de cada uno de ellos y los niveles de interdependencia que pudiera haber entre los mismos. Se analizaron sus aspectos poblacionales, imagen urbana, nivel de servicios y equipamientos de los principales núcleos poblacionales de Tela: Tela, Mezapa, Buenos Aires 1, El Guano, Triunfo de la Cruz, La Fortuna, Paujiles, San Alejo, San Juan, Toloa Adentro, Tornabé. Este sistema de ciudades permite observar pequeños poblados rurales. En este apartado se analizará el sistema de ciudades del municipio y las interrelaciones que se generan entre los poblados. Cabe destacar que en la selección de los principales poblados también participaron los técnicos municipales, pues son ellos que conocen las características de cada poblado para que sean considerados relevantes dentro del municipio.

Se localizaron 31 asentamientos humanos; los cuales al año 2001 contaban, según el Censo de Población y Vivienda del Instituto Nacional de Estadísticas de Honduras 2001, con los siguientes datos de población. 
Tabla 1: Datos de población principales asentamientos humanos municipio de Tela

\begin{tabular}{|c|c|}
\hline \multicolumn{2}{|c|}{ Asentamientos humanos municipio de Tela } \\
\hline Asentamiento humano & Población \\
\hline Tela & 27.990 \\
\hline Agua Chiquita & 524 \\
\hline Buena Vista & 696 \\
\hline Buenos Aires I & 1.129 \\
\hline Los Laureles & 907 \\
\hline El Barro o Buena Vista & 739 \\
\hline El Cedro & 503 \\
\hline El Guano & 1.107 \\
\hline El Junco & 569 \\
\hline El Jute & 823 \\
\hline Triunfo de la Cruz & 2.032 \\
\hline Kilómetro 13 & 809 \\
\hline Lancetilla & 856 \\
\hline La Esperanza & 621 \\
\hline La Fortuna & 1.086 \\
\hline La Ica & 846 \\
\hline La Yusa & 772 \\
\hline Las Metalias & 730 \\
\hline Las Quebradas & 808 \\
\hline Mezapa & 5.919 \\
\hline Morazán & 516 \\
\hline Nombre de Dios & 587 \\
\hline Paujiles & 1.209 \\
\hline Planes & 689 \\
\hline Rio Tinto & 597 \\
\hline San Alejo & 2.107 \\
\hline San Juan & 2.114 \\
\hline Santiago & 878 \\
\hline Toloa Adentro & 1.494 \\
\hline Tornabé & 1.529 \\
\hline Zoilabe & 683 \\
\hline
\end{tabular}

Fuente: Elaboración propia, en base a datos del Censo de Población y Vivienda, INE, 2001. 


\section{EL SISTEMA DE ASENTAMIENTOS HUMANOS URBANOS Y RURALES}

Estos 31 asentamientos humanos fueron evaluados y como resultado de esta evaluación se ha otorgado a cada uno diferentes niveles de relevancia municipal. Se genera la siguiente tabla:

Tabla 2: Sistema de asentamientos humanos municipio de Tela

\begin{tabular}{|c|c|c|c|c|c|c|}
\hline $\begin{array}{c}\text { Asentamiento } \\
\text { humano }\end{array}$ & $\begin{array}{c}\text { Pobla- } \\
\text { ción 70\% }\end{array}$ & $\begin{array}{c}\text { Imagen } \\
\text { urbana 10\% }\end{array}$ & $\begin{array}{c}\text { Accesibili- } \\
\text { dad 10\% }\end{array}$ & $\begin{array}{c}\text { Equipamien- } \\
\text { tos y servi- } \\
\text { cios 10\% }\end{array}$ & Total & $\begin{array}{c}\text { Nivel/ } \\
\text { Catego- } \\
\text { ría }\end{array}$ \\
\hline Tela & 70 & 7.5 & 10 & 10 & 97.5 & 1 \\
\hline Mezapa & 56 & 5 & 5 & 5 & 71 & 2 \\
\hline $\begin{array}{c}\text { Triunfo de la } \\
\text { Cruz }\end{array}$ & 42 & 5 & 10 & 5 & 62 & 3 \\
\hline Tornabé & 42 & 5 & 10 & 5 & 62 & 3 \\
\hline El Guano & 42 & 0 & 10 & 5 & 57 & 3 \\
\hline Paujles & 42 & 0 & 10 & 5 & 57 & 3 \\
\hline San Juan & 42 & 0 & 10 & 5 & 57 & 3 \\
\hline $\begin{array}{c}\text { Buenos } \\
\text { Aires 1 }\end{array}$ & 42 & 0 & 5 & 5 & 52 & 3 \\
\hline San Alejo & 42 & 0 & 10 & 0 & 52 & 3 \\
\hline Toloa Adentro & 42 & 0 & 0 & 5 & 47 & 3 \\
\hline El Jute & 28 & 0 & 10 & 5 & 43 & 4 \\
\hline Las Metalias & 28 & 0 & 10 & 5 & 43 & 4 \\
\hline Santiago & 28 & 0 & 10 & 5 & 43 & 4 \\
\hline Zoilabe & 28 & 0 & 10 & 5 & 43 & 4 \\
\hline La Fortuna & 42 & 0 & 0 & 0 & 42 & 4 \\
\hline El Junco & 28 & 0 & 10 & 0 & 38 & 4 \\
\hline Kilómetro 13 & 28 & 0 & 5 & 5 & 38 & 4 \\
\hline Lancetilla & 28 & 0 & 10 & 0 & 38 & 4 \\
\hline La Esperanza & 28 & 0 & 5 & 5 & 38 & 4 \\
\hline Planes & 28 & 0 & 5 & 5 & 38 & 4 \\
\hline Buena Vista & 28 & 0 & 5 & 0 & 33 & 4 \\
\hline Los Laureles & 28 & 0 & 0 & 5 & 33 & 4 \\
\hline El Cedro & 28 & 0 & 5 & 0 & 33 & 4 \\
\hline
\end{tabular}




\begin{tabular}{|c|c|c|c|c|c|c|}
\hline $\begin{array}{c}\text { Asentamiento } \\
\text { humano }\end{array}$ & $\begin{array}{c}\text { Pobla- } \\
\text { ción 70\% }\end{array}$ & $\begin{array}{c}\text { Imagen } \\
\text { urbana 10\% }\end{array}$ & $\begin{array}{c}\text { Accesibili- } \\
\text { dad 10\% }\end{array}$ & $\begin{array}{c}\text { Equipamien- } \\
\text { tos y servi- } \\
\text { cios 10\% }\end{array}$ & Total & $\begin{array}{c}\text { Nivel/ } \\
\text { Catego- } \\
\text { ría }\end{array}$ \\
\hline La Ica & 28 & 0 & 0 & 5 & 33 & 4 \\
\hline Morazán & 28 & 0 & 0 & 5 & 33 & 4 \\
\hline Agua Chiquita & 28 & 0 & 0 & 0 & 28 & 4 \\
\hline El Barro & 28 & 0 & 0 & 0 & 28 & 4 \\
\hline La Yusa & 28 & 0 & 0 & 0 & 28 & 4 \\
\hline $\begin{array}{c}\text { Las Quebra- } \\
\text { das }\end{array}$ & 28 & 0 & 0 & 0 & 28 & 4 \\
\hline $\begin{array}{c}\text { Nombre de } \\
\text { Dios }\end{array}$ & 28 & 0 & 0 & 0 & 28 & 4 \\
\hline Rio Tinto & 28 & 0 & 0 & 0 & 28 & 4 \\
\hline
\end{tabular}

Fuente: Elaboración propia en base a datos del INE, visitas de campo y encuestas a técnicos municipales.

\section{Catalogación según los niveles}

Nivel 1: Inician su independencia socioeconómica, su área de influencia es inter-departamental, dependen de las ciudades de mayor jerarquía nacional, constituye polo de desarrollo departamental.

Nivel 2: Cuentan con servicios básicos mínimos, su jerarquía es local, al interior del municipio, dependen en el alto grado de otras ciudades con más jerarquía, generalmente existentes dentro de su municipio. Es el primer nivel para que un asentamiento humano rural, empiece a presentar características para ser considerado como centro urbano.

Nivel 3: Cuentan con algún servicio básico y de forma parcial, sus actividades económicas y comerciales son básicamente agrícolas. Representan, según censo del 2001 , el $13 \%$ de los asentamientos humanos del país. Algunos técnicos los denominan pre-urbanos. Su rango inicia con los 501 habitantes. Son asentamientos que presentan una definida estructura física y cuentan una base organizativa comunitaria. 
Nivel 4: Cuentan con gran importancia los servicios básicos, siguen siendo dependientes, pero tienen alguna jerarquía a nivel rural local. Gozan de una organización comunal sólida, sin embargo hay actividades que ofrecen empleo temporal. La migración es menor y cuentan con seguridad con escuela primaria completa y un centro de salud rural (CESAR).

\section{CLASIFICACIÓN POTENCIAL DE LOS ASENTAMIENTOS HUMANOS EN FUNCIÓN DE LIMITANTES AL DESARROLLO URBANO}

En la matriz que se presenta a continuación se enumeran los asentamientos humanos jerarquizados en el sistema de ciudades del municipio y clasificados en función de sus limitantes al desarrollo urbano. 
TABLA 3 clasificación potencial de los asentamientos humanos en función de limitantes al desarrollo urbano

\begin{tabular}{|c|c|c|c|c|c|c|c|c|}
\hline $\begin{array}{c}\text { Asentamiento } \\
\text { Humano }\end{array}$ & Población & $\begin{array}{c}\text { Jerarquía } \\
\text { Urbana }\end{array}$ & $\begin{array}{c}\text { Mérito de } \\
\text { Conservación }\end{array}$ & $\begin{array}{c}\text { Área } \\
\text { Inundable }\end{array}$ & Pendientes & \begin{tabular}{|c|} 
Areas \\
Protegidas
\end{tabular} & Valor & Clasificación \\
\hline El Guano & 1.107 & 3 & 1 & 1 & 1 & 1 & 4 & Incentivar \\
\hline Buenos Aires 1 & 1.129 & 3 & 1 & 1 & 1 & 1 & 4 & Incentivar \\
\hline San Alejo & 2.107 & 3 & 1 & 1 & 1 & 1 & 4 & Incentivar \\
\hline El Jute & 823 & 4 & 1 & 1 & 1 & 1 & 4 & Incentivar \\
\hline Las Metalias & 730 & 4 & 1 & 1 & 1 & 1 & 4 & Incentivar \\
\hline Santiago & 878 & 4 & 1 & 1 & 1 & 1 & 4 & Incentivar \\
\hline Zoilabe & 683 & 4 & 1 & 1 & 1 & 1 & 4 & Incentivar \\
\hline La Fortuna & 1.086 & 4 & 1 & 1 & 1 & 1 & 4 & Incentivar \\
\hline El Junco & 569 & 4 & 1 & 1 & 1 & 1 & 4 & Incentivar \\
\hline Kilòmetro 13 & 809 & 4 & 1 & 1 & 1 & 1 & 4 & Incentivar \\
\hline La lca & 846 & 4 & 1 & 1 & 1 & 1 & 4 & Incentivar \\
\hline El Barro & 739 & 4 & 1 & 1 & 1 & 1 & 4 & Incentivar \\
\hline La Yusa & 772 & 4 & 1 & 1 & 1 & 1 & 4 & Incentivar \\
\hline Tela & 27.99 & 1 & 1 & 2 & 1 & 1 & 5 & Consolidar \\
\hline Triunfo de la Cruz & 2.032 & 3 & 3 & 2 & 1 & 1 & 7 & Consolidar \\
\hline Paujiles & 1.209 & 3 & 1 & 4 & 1 & 1 & 7 & Consolidar \\
\hline Planes & 689 & 4 & 1 & 4 & 1 & 1 & 7 & Consolidar \\
\hline Agua Chiquita & 524 & 4 & 1 & 1 & 4 & 1 & 7 & Consolidar \\
\hline Mezapa & 5.919 & 2 & 1 & 5 & 1 & 1 & 8 & Consolidar \\
\hline Toloa Adentro & 1.494 & 3 & 1 & 5 & 1 & 1 & 8 & Consolidar \\
\hline Los Laureles & 907 & 4 & 1 & 5 & 1 & 1 & 8 & Consolidar \\
\hline Tornabè & 1.529 & 3 & 4 & 2 & 1 & 3 & 10 & Consolidar \\
\hline San Juan & 2.114 & 3 & 4 & 1 & 1 & 4 & 10 & Consolidar \\
\hline Morazàn & 516 & 4 & 4 & 1 & 5 & 1 & 11 & Desincentivar \\
\hline Las Quebradas & 808 & 4 & 4 & 1 & 5 & 1 & 11 & Desincentivar \\
\hline Nombre de Dios & 587 & 4 & 4 & 1 & 5 & 1 & 11 & Desincentivar \\
\hline Buena Vista & 696 & 4 & 4 & 1 & 5 & 1 & 11 & Desincentivar \\
\hline El Cedro & 503 & 4 & 4 & 1 & 5 & 1 & 11 & Desincentivar \\
\hline Lancetilla & 856 & 4 & 5 & 1 & 1 & 5 & 12 & Desincentivar \\
\hline La Esperanza & 621 & 4 & 4 & 1 & 5 & 4 & 14 & Desincentivar \\
\hline Rio Tinto & 597 & 4 & 5 & 5 & 1 & 5 & 16 & Desincentivar \\
\hline
\end{tabular}

Fuente: Elaboración propia en base a datos obtenidos en visitas de campo,

Simbología

y análisis de ortofotos 2012

5: Muy Alta, 4: Alta, 3: Media, 2: Baja, 1: Muy Baja 
En la matriz se puede observar, que los asentamientos humanos: Morazán, Las Quebradas, Nombre de Dios, Buena Vista, El Cedro, Lancetilla, La Esperanza, y Río Tinto, son asentamientos a desincentivar debido a que tienen una considerable área inundable, y por ende un muy alto riesgo a inundación y pendientes elevadas, lo que los hace asentamientos humanos poco sostenibles y no viables.

Los asentamientos de Tela, Triunfo de la Cruz, Paujiles, Planes, Agua Chiquita, Mezapa, Toloa Adentro, Los Laureles, Tornabé y San Juan, se ubican como asentamientos a consolidar, sin posibilidades a un crecimiento importante, debido a las diversas condicionantes que se ubican en sus proximidades.

Mientras que los asentamientos de El Guano, Buenos Aires 1, San Alejo, El Jute, Las Metalias, Santiago, Zoilabe, La Fortuna, El Junco, Kilómetro 13, La Ica, El Barro, La Yusa son los que precisan ser incentivados en el municipio de Tela (desde el punto de vista de condicionantes naturales al desarrollo urbano) debido a que cuentan con las condiciones territoriales para expandir su crecimiento futuro. Esta matriz constituye el punto de partida para la siguiente etapa de la investigación: la prospectiva.

Figura 1: Asentamientos humanos municipio de Tela, clasificados según sus condicionantes al desarrollo urbano

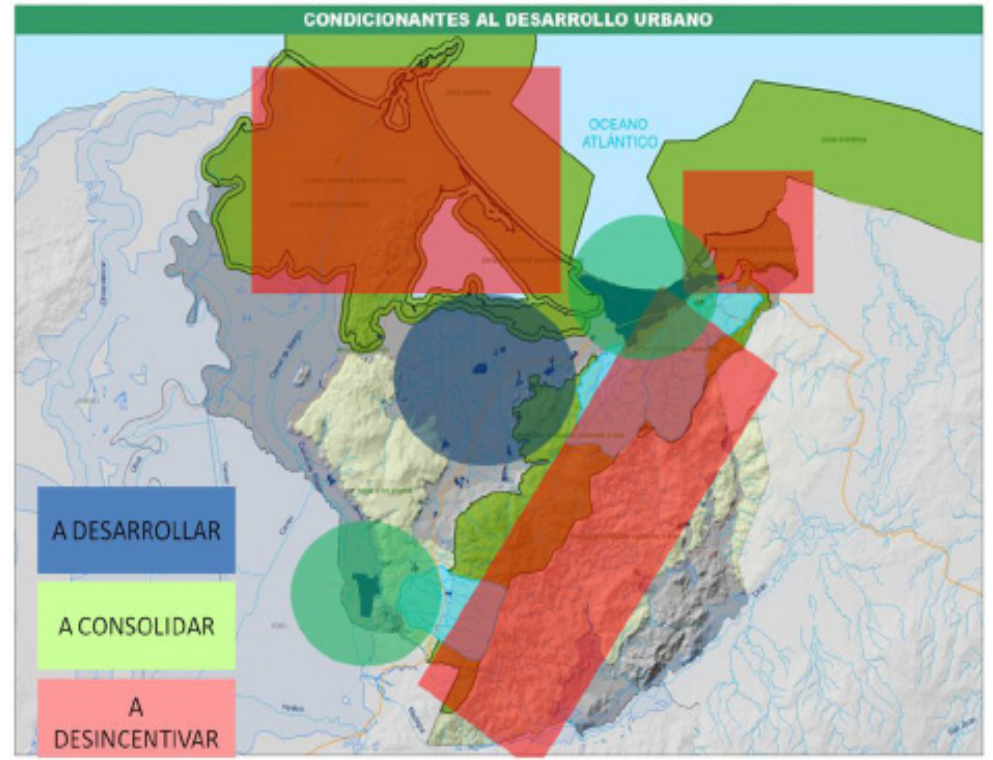

Fuente: Elaboración propia en base a datos obtenidos en análisis de ortofotos, 2012. 


\section{CONCLUSIONES}

- La fase de prospectiva es la fase más compleja y peor desarrollada en los planes de ordenamiento territorial de la región centroamericana. Se considera que en la actualidad existe: omisión, diferencia de planteamientos, errores conceptuales, desintegración, y falta de representación en mapas, entre otros lo cual se trata de mejorar en la metodología planteada.

- La metodología diseñada plantea un método integral y científico para abordar la fase de prospectiva en los planes de ordenamiento territorial y en especial para casos centroamericanos. Esta metodología se puede entender como un metamodelo, es decir, un modelo general formado por submodelos específicos: población, ambiente, infraestructuras y económico. El metamodelo establece un orden de prelación entre los submodelos. No se recomienda la alteración de este orden pues el resultado será distinto y erróneo. Para la elaboración de los submodelos se usan diferentes herramientas de proyección de variables; algunas de estas han sido diseñadas por investigadores, otras se diseñan en esta tesis.

- Una de las claves del éxito de la prospectiva estriba en una buena definición de las hipótesis territoriales. Sin ello, los escenarios serán poco reales y no servirán para definir una imagen objetivo aplicable; se considera la consulta a grupos de expertos a través de metodologías como el método Delphi o El Ábaco de Regnier.

\section{BIBLIOGRAFÍA}

- Alberti, M. y Bettini, V. (1998). "Sistemas urbanos e indicadores de sostenibilidad", en Elementos de ecología urbana. (V. Bettini). Madrid: Trotta.

- Allende J. (1995). "Desarrollo sostenible. De lo global a lo local" en Ciudad y territorio. Estudios Territoriales, № 104, pp. 267-281.

- Aymonino, C. (1981). El significado de las ciudades. Madrid.

- Bosque Sendra, J. y Moreno Jiménez, A. (1990). "Facility location analysis and planning: An EGIS approach" First Europoean Conference on Geographic Information Systems, pp. 87-94. Utrecht: EGIS Foundation. 
- Brunet, R. (1980). "La composición de los modelos en el análisis espacial". L'espace Geographique, pp. 253-265.

- Camagni Roberto (1999) "El desarrollo urbano sostenible. Razones y fundamentos de un programa de investigación" en Papeles de Economía Española No 80, pp. 266-290.

- Chueca Goitia, F. (1998). Breve historia del urbanismo. Madrid: Alianza Editorial.

- Comisión Europea (1998). "Manual sobre evaluación ambiental de planes de desarrollo regional y programas de los fondos estructurales de UE". Anexo IV: Indicadores. Dirección General XI, Medio Ambiente.

- Gabellini, P. (2001). "Planificación del territorio y del ambiente". Técnicas urbanísticas, Roma: Carocci.

- Godet Michael (2007). Prospectiva estratégica: problemas y métodos. Francia: Editorial Donostia.

- Gómez Orea (2007) Ordenación territorial. Madrid: Editorial Mundi.

- Haggett, P. (1988). Geografía, una síntesis moderna. Barcelona: Omega.

- Harris, C.D. (1975). Modelos de desarrollo urbano. Barcelona: OIKOS-TAU.

- Harris, C.D. (1998). "Diffusion of urban models: a case study", Urban Geography $\mathrm{N}^{0} 19, \mathrm{p} .49$.

- Inypsa, Diagnóstico territorial municipio de Tela Honduras. Tegucigalpa, Honduras 2011.

- Lynch, Kevin (1998). La imagen de la ciudad. México: Editorial Gustavo Gili

- Maza Vázquez, F. (2003-2004). Planeamiento urbanístico en el corredor de Henares de Guadalajara: una perspectiva desde el desarrollo sostenible. España: Universidad de Alcalá.

- Maza Vázquez, F. (2007). Evolución espacial de Guadalajara, según la cartografía. España: Patronato de Cultura del Ayuntamiento de Guadalajara. 
- Rossi, A. (1995). La arquitectura de la ciudad. México: Editorial Gustavo Gili

- Rubio Blanco (2012). Prospectiva en el ordenamiento territorial. Tegucigalpa.

- Wingo, L. (1976). Ciudades y espacio. Barcelona: OIKOS-TAU. 